\title{
Oxidation of Si surface by a pulsed Nd : YAG laser
}

\author{
G Aygun ${ }^{1,5}$, E Atanassova ${ }^{2}$, A Alacakir ${ }^{3}$, L Ozyuzer $^{4}$ and \\ R Turan ${ }^{1}$ \\ ${ }^{1}$ Department of Physics, Middle East Technical University, Ankara, Turkey \\ ${ }^{2}$ Institute of Solid State Physics, Bulgarian Academy of Sciences, Sofia 1784, Bulgaria \\ ${ }^{3}$ Ankara Nuclear Research \& Training Center, Ankara, Turkey \\ ${ }^{4}$ Department of Physics, Izmir Institute of Technology, Izmir, Turkey \\ E-mail: aygun@newton.physics.metu.edu.tr
}

Received 27 March 2004

Published 12 May 2004

Online at stacks.iop.org/JPhysD/37/1569

DOI: $10.1088 / 0022-3727 / 37 / 11 / 011$

\begin{abstract}
$\mathrm{SiO}_{2}$ thin films have been obtained by $1064 \mathrm{~nm} \mathrm{Nd}$ : YAG laser oxidation of $\mathrm{p}$-Si in the presence of $\mathrm{O}_{2}$. The thickness uniformity, dielectric and electrical properties of the layers have been studied. The effect of both the laser beam energy density and the substrate temperature on the oxide growth is also discussed. It was established that there exists an interval of laser beam energy density in which the oxidation occurs without surface melting. The oxidation process is controlled by the laser beam energy density rather than by the substrate temperature (673-748 K) and the higher laser power results in a thicker oxide. X-ray photoelectron spectroscopy (XPS) was used to provide information on the oxide composition. XPS results revealed that the as-grown oxide is a mixed layer of $\mathrm{SiO}_{2}$ and $\mathrm{Si}_{2} \mathrm{O}$, which are distributed nonuniformly through the depth. MOS capacitors fabricated on the grown oxide exhibited typical capacitance-voltage, conductance-voltage characteristics. However, the density of interface states and oxide charge density were found to be higher than the typical values of thermally grown oxides. The quality of the oxide layers can be further improved by optimization of the process parameters and/or by post-processing of the grown films. It is concluded that the $\mathrm{SiO}_{2}$ films formed by the technique of $\mathrm{Nd}$ : YAG laser-enhanced oxidation at low temperature are potentially useful for device applications.
\end{abstract}

\section{Introduction}

One of the most important issues in modern microelectronics is the fabrication of dielectric layers with enhanced capacitance properties to meet the requirements of the high density dynamic random access memories. There are two main directions in the research activities concerning the production of these films: (i) the fabrication of ultra-thin and electrically reliable $\mathrm{SiO}_{2}$ layers on the $\mathrm{Si}$ substrate [1] and (ii) the fabrication of alternative dielectrics with high dielectric constant (high- $\kappa$ materials) [2-4]. In both cases, however, there is always great interest in novel processing techniques that may, in

5 Author to whom any correspondence should be addressed. some way, improve the existing methods of production. Formation of an oxide layer on $\mathrm{Si}$ substrate can be realized by a number of well-known techniques, such as thermal oxidation, a variety of chemical vapour deposition methods, sputtering, plasma assisted oxidation and oxidation by pulsed or continuous light sources [1]. Local oxidation by atomic force microscopy (AFM) appears to be another novel technique which has recently received much attention [5]. Among all these techniques, laser assisted oxidation has attracted special attention due to its ability to induce localized oxidation with good spatial resolution and its ability to achieve a good control over the thickness of very thin layers of 1-2 nm at low processing temperatures [6-10]. Various laser sources 
$\left(\mathrm{Ar}^{+}\right.$[7], $\mathrm{CO}_{2}$ [8], ruby [9], excimer [10]) with continuous or pulsed beams have been used to process $\mathrm{Si}$ or metal surfaces for the oxidation, and successful growth of thin oxide layers has been demonstrated. A pulsed Nd: YAG laser operating at $1064 \mathrm{~nm}$ has recently been used for oxidation of metal surfaces to obtain high $-\kappa$ dielectrics [11]. The emitted laser energy is $1.165 \mathrm{eV}$ at $1064 \mathrm{~nm}$, which is slightly more than the bandgap energy of $\mathrm{Si}$ crystal; the absorption by the $\mathrm{Si}$ surface is then expected to be weak due to the limiting value of the photon energy. On the other hand, high peak energy pulses of the $\mathrm{Nd}$ : YAG laser beam usually result in melting of the surface [12]. The effect of laser pulses on the Si surface morphology and the mechanisms of the melting were reported $[12,13]$. So far, observation of high quality $\mathrm{SiO}_{2}$ on $\mathrm{Si}$ grown by $\mathrm{Nd}$ : YAG laser has not been reported. The question here is, whether or not an efficient oxidation can be achieved without causing an undesirable deformation of the surface. If the answer is yes, then the feasibility of $\mathrm{SiO}_{2}$ formation and device characteristics with this kind of oxide layer needs to be investigated. In this paper, we report on the formation of a thin $\mathrm{SiO}_{2}$ layer on an $\mathrm{Si}$ surface by a pulsed Nd: YAG laser scanned over a predefined region, and demonstrate the successful oxidation of the $\mathrm{Si}$ surface with a laser power that is slightly lower than that needed for surface melting. Special attention is paid to the influence of both laser power and the substrate temperature on the oxidation process and thickness uniformity of the layers obtained. For some of the films, the elemental composition and the chemical bonding were analysed by means of x-ray photoelectron spectroscopy (XPS). The electrical properties of the films in the metal-oxide-Si (MOS) capacitor configuration were also examined.

\section{Experimental procedure}

The Si substrates were p-type (100) oriented wafers with a resistivity of $15-17 \Omega \mathrm{cm}$. The samples were cleaned chemically in boiling trichloroethylene and acetone together with an ultrasonic bath of ultra-pure water. They were dipped into diluted $\mathrm{HF}$ for a few seconds just before loading them into the chamber. The surface of films was hydrophobic after the HF dip showing that the native oxide layer was removed from the surface. They were mounted onto the substrate holder of the chamber which was evacuated to a pressure below $0.133 \mathrm{~Pa}$. The holder was preheated to temperatures between 673 and $748 \mathrm{~K} . \mathrm{O}_{2}$ gas was introduced into the chamber (working gas pressure of $123 \mathrm{~Pa}$ ) after reaching the desired substrate temperature, $T_{\mathrm{s}}$. The laser treatment was carried out using Nd: YAG radiation at $1064 \mathrm{~nm}$ (EKSPLA Pulsed Laser NL301). The laser parameters are summarized in table 1 . The laser beam has a hat-top intensity profile and thermal effects are expected at the irradiated surface [6,14]. Preliminary experiments showed that an oxidation time of at least $20 \mathrm{~min}$

Table 1. Laser parameters.

\begin{tabular}{llllll}
\hline Wavelength & $\begin{array}{l}\text { Pulse } \\
\text { duration }\end{array}$ & $\begin{array}{l}\text { Pulse } \\
\text { frequency }\end{array}$ & $\begin{array}{l}\text { Total } \\
\text { pulse } \\
\text { energy }\end{array}$ & $\begin{array}{l}\text { Near field } \\
\text { intensity } \\
\text { profile }\end{array}$ & $\begin{array}{l}\text { Beam } \\
\text { radius }\end{array}$ \\
\hline $1064 \mathrm{~nm}$ & $4.7 \mathrm{~ns}$ & $10 \mathrm{~Hz}$ & $320 \mathrm{~mJ}$ & Hat top & $3 \mathrm{~mm}$ \\
\hline
\end{tabular}

was needed to be able to obtain a measurable oxide layer on $\mathrm{Si}$. That is why, in this study, we used a constant exposure time duration of $30 \mathrm{~min}$ in order to obtain a thick enough film so that its properties could be successfully characterized. The main laser fluence varies in the range between 2.65 and $3.51 \mathrm{~J} \mathrm{~cm}^{-2}$ per pulse. The schematic illustration of the experimental apparatus is shown in figure 1 . Having attached to a $\mathrm{X}-\mathrm{Y}$ scanner system, a $3 \mathrm{~cm}$ converging lens with a focal length of approximately $25 \mathrm{~cm}$ generated a spot of diameter $1.8 \mathrm{~mm}$ on the substrate. The $\mathrm{X}-\mathrm{Y}$ scanner system was controlled by a computer via its parallel port to direct the laser beam on a certain region with controllable dimensions. By means of the scanner system, the laser light was made to shine on a region on the substrate of area approximately $4 \times 4 \mathrm{~mm}^{2}$ and this area can be reduced or increased by software. The oxide thickness, $d$, and the refractive index, $n$, of the oxide layers obtained were determined by an ellipsometer, $(\lambda=632.8 \mathrm{~nm})$. The electrical characterization of the layers was carried out using MOS capacitors with evaporated $\mathrm{Al}$ gate electrodes with an area of $1.96 \times 10^{-3} \mathrm{~cm}^{2}$. The back of the wafer was HF-cleaned and was followed by $\mathrm{Al}$ metallization. The dielectric and electrical characteristics of the layers were studied using capacitance-voltage $(C-V)$, conductance-voltage $(G-V)$, and current-voltage $(I-V)$ measurements. The oxide charge $Q_{\mathrm{f}}$ was evaluated from the flat band voltage $V_{\mathrm{fb}}$ of the highfrequency ( $1 \mathrm{MHz}) C-V$ curves. XPS data were obtained in an ESCALABII high vacuum apparatus (VG Scientific Ltd) with a residual gas pressure better than $1 \times 10^{-8} \mathrm{~Pa}$. $\mathrm{AlK}_{\alpha}$ $(1486.6 \mathrm{eV})$ radiation was used to excite the photoelectrons from the sample. All spectra were taken at $300 \mathrm{~K}$. The photoelectrons were separated by a semi-spherical analyser with a pass energy of $10 \mathrm{eV}$. The instrumental resolution measured as the full-width at half-maximum (FWHM) of the Ag $3 d_{5 / 2}$ photoelectron peak was $1.0 \mathrm{eV}$. The photoelectron lines of Si $2 p$ and $\mathrm{O} 1 \mathrm{~s}$ were recorded. The energy position of the peaks was determined with an accuracy of $0.1 \mathrm{eV}$. The spectra were obtained under an angle of observation of the photoemitted electrons of $\theta=90^{\circ}$ (with respect to the surface plane).

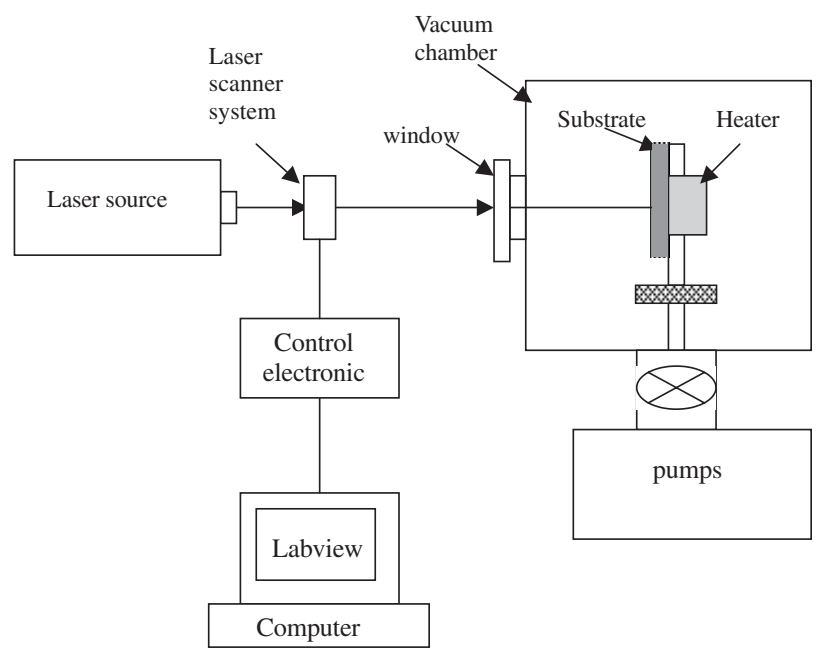

Figure 1. Schematic illustration of the laser system. 

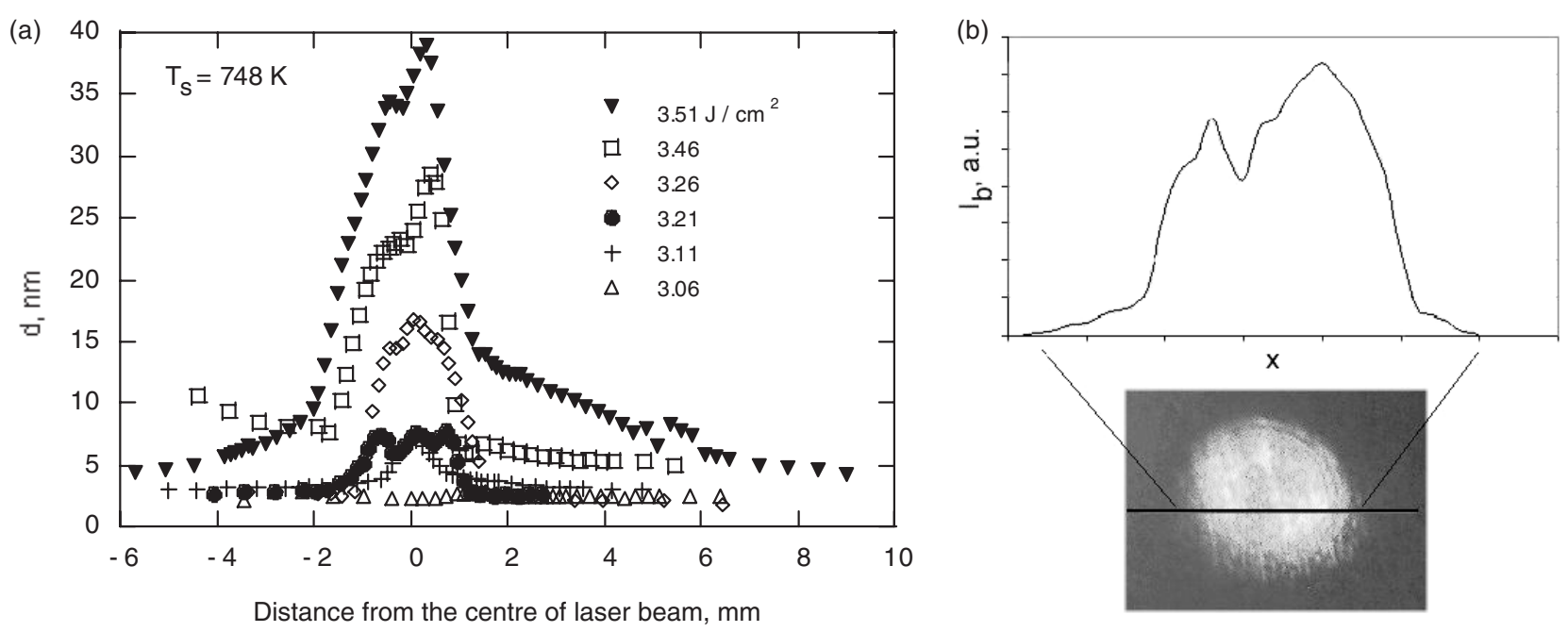

Figure 2. (a) Film thickness distribution profile with respect to the centre of the laser beam for different beam energy fluences $\left(T_{\mathrm{s}}=748 \mathrm{~K}\right)$. (b) Intensity profile of the laser spot along the scanning direction. Degree of darkness corresponds to the intensity of energy fluence, i.e. the lighter the grey colour is, the more intensive the energy fluence is.

\section{Results and discussions}

\subsection{Thickness uniformity}

3.1.1. Effect of laser beam energy density. The variations of the oxide thickness as measured ellipsometrically over the exposed area for different laser beam energy densities (for simplicity we will also use the term laser power) are shown in figure 2(a) (the substrate temperature is constant at $748 \mathrm{~K}$ ). The highest value of oxide thickness, $d$, is at the centre of the laser beam, and the thickness is approximately uniform across the laser irradiated region. As can be seen, a relatively large region around the active spot is also oxidized (two tails of Gaussian curves) but with significantly reduced oxide thicknesses. The measured thicknesses over the periphery regions are fairly similar for a certain value of power, and the effect is more pronounced for lower powers. Here and later we will focus only on the region close to the centre of the laser beam (denoted by ' 0 ' in figure $2(a)$ ), and its profile evaluation with the laser power, $P$. The oxidation process is not detected at all for the fluence less than about $3.00 \mathrm{~J} \mathrm{~cm}^{-2}$ and the oxide detected is virtually native oxide on the wafer, slightly affected also by the substrate temperature. The start of active oxidation was detected for $P \geqslant 3.00 \mathrm{~J} \mathrm{~cm}^{-2}$ and the beam energy density level significantly affects the oxide thickness at the centre of the oxidized region, $d$ being lower for lower laser powers and higher for higher powers. The oxide thickness at the centre of the beam, ranging from 7 to $34 \mathrm{~nm}$, is proportional to $P$ from 3.06 to the highest value $\left(3.51 \mathrm{~J} \mathrm{~cm}^{-2}\right)$, i.e. the higher laser power results, essentially, in sufficiently thicker films, and the growth takes place at a faster rate. However, a nonuniformity of the oxidation in a wide range around the beam centre is obvious. Nevertheless, the thickness profile shows that a relatively flat narrow range can be found over the distance of $1 \mathrm{~mm}$ from the centre across which the thickness variation is within $10 \%$. This dispersion is lowest for the highest $T_{\mathrm{s}}$ and $P$, i.e. it changes between $3 \%$ and $4 \%$ at $T_{\mathrm{s}}=748 \mathrm{~K}$ while it is around $10 \%$ for the lowest $T_{\mathrm{s}}$. The area of this active oxidized spot on the wafer is in fact independent of the laser power. It should be noted that, for the higher energy density, a second peak appears in the oxidant profile, close to the main one and corresponding to slightly higher oxide thickness. The right-hand side of figure 2(a) illustrates this peak. Even though there may be few reasons for this second peak, the variation in the oxide thickness on the wafer is most likely caused by the nonuniformity in the laser beam due to the intra-cavity components of the laser, which leads to the hat-top shape. To check this, the two-dimensional intensity profile of the beam was recorded by a high resolution CCD camera. The processing of the image demonstrates that it is nonuniform and includes two main peaks, one of which is more intensive than the other. The profile is shown in figure $2(b)$. While this can be an explanation for the nonuniformity of the processed area, the small step scanning of the beam on the wafer homogenizes the exposed area in the case of a perfect X-Y scanner. Since the converging lens moves relative to the laser beam, the focal point also moves resulting in the intensity variation. We generally obtain an oxidized region having more affected area at one corner of the oxidized area, despite the fact that the exact intensity distribution is unknown. This second peak's region on the wafer is not crashed or melted and cannot be seen by the naked eye, but it can be detected from the ellipsometer measurements. So, the results imply that this feature of the thickness profile can be attributed to the nonuniformity of the intensity of the laser beam rather than to the mechanism of laser oxidation itself. Additional evidence for this conclusion comes from the relatively good agreement between the shape of the two profiles, the beam intensity distribution and the thickness distribution. In other words, the second profile roughly repeats the former.

Figure 3 shows the oxide thickness, $d$, in the vicinity of the main peak of the thickness distribution profile as a function of the laser beam energy density for various $T_{\mathrm{s}}$. The behaviour of the curves shows that the laser beam energy significantly affects the oxidation process, and the data can be described by a linear-parabolic function for all $T_{\mathrm{s}}$ values. It is also apparent from the figure that the oxidation process effectively starts when the laser energy fluence exceeds $\sim 3.00 \mathrm{~J} \mathrm{~cm}^{-2}$. 


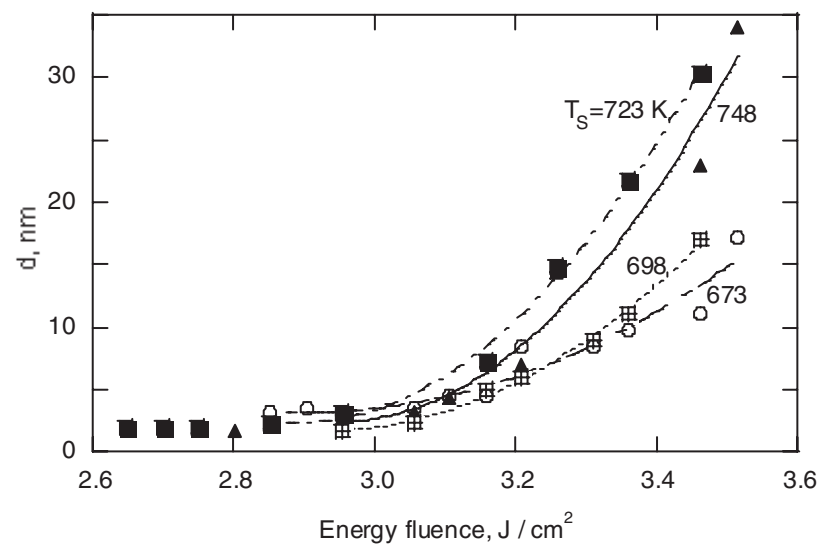

Figure 3. Oxide thickness as a function of laser fluence for different $T_{\mathrm{s}}$.

The energy density 'threshold' is virtually independent of substrate temperature, suggesting that the overall oxidation is controlled (as is expected) by the laser fluence rather than $T_{\mathrm{s}}$. It should be emphasized that the laser oxidation in our case begins in fact from an initial oxide with a thickness of $\sim 2-3 \mathrm{~nm}$ formed on the surface of heated wafer under the action of active oxidizing ambient during the time elapsed between $\mathrm{O}_{2}$ gas introduction and laser treatment. The oxide thickness measured for $P \leqslant 3.00 \mathrm{~J} \mathrm{~cm}^{-2}$ (figure 3) corresponds to the thickness of this native oxide, which is slightly influenced by $T_{\mathrm{s}}$. For $P$ above $\sim 3.00 \mathrm{~J} \mathrm{~cm}^{-2}$, the oxide grows relatively fast. At the other extreme point, where the laser energy density exceeds $3.60 \mathrm{~J} \mathrm{~cm}^{-2}$, the catastrophic deformation of the surface due to the melting is observed. As a consequence, the existence of a laser energy density interval is detected (namely between 3.00 and $3.60 \mathrm{~J} \mathrm{~cm}^{-2}$ ) where the oxidation takes place without causing any structural deformation of the surface. The results indicate that by varying the energy beam density it is possible to modulate the oxide thickness in an appropriate controlled manner during the growth process. With increasing beam density, we observed stable film formation for all substrate temperatures, and both higher $P$ and higher $T_{\mathrm{s}}$ produce a thicker film. It is obvious that when the substrate temperature is higher the oxide is thicker, at a constant laser beam energy density, i.e. at certain values of $P$ the thicker oxides are grown at higher $T_{\mathrm{s}}$. Even though this qualitative conclusion can be drawn on the effect of temperature dependence, a quantitative analysis is hardly possible due to the unknown chemical and physical processes taking place during the oxidation and also the scattered nature of the data points.

3.1.2. Effect of substrate temperature. It is generally accepted that the oxidation of a pure Si surface by the laser beam occurs via heating of the surface locally by a laser beam. The effect of heating is two-fold: increasing the reaction rate of $\mathrm{Si}$ atoms with $\mathrm{O}$ atoms and increasing the number of free carriers in the surface region, which in turn enhances the free carrier absorption. The oxidation rate is then expected to depend on the substrate temperature. The impact of $T_{\mathrm{s}}$ on $d$ for two representative substrate temperatures, from the 'lower' and 'higher' $T_{\mathrm{s}}$ regions, namely 673 and $748 \mathrm{~K}$, is shown

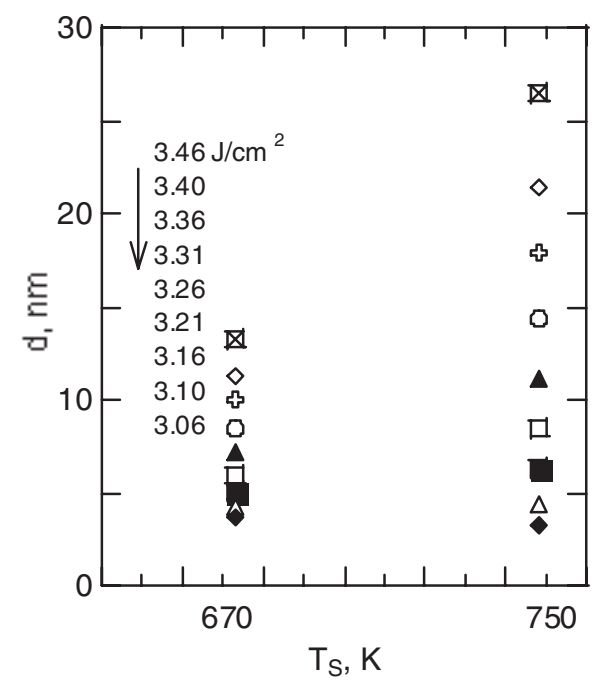

Figure 4. Oxide thickness as a function of $T_{\mathrm{s}}$, for various laser beam energy fluences.

in figure 4. As can be seen, with an enhancement of $T_{\mathrm{s}}, d$ generally increases but the degree of the increase significantly depends on the laser energy density: no thickness variation with $T_{\mathrm{S}}$ is detected up to the energy density 'threshold' for real oxidation $\left(P \sim 3.00 \mathrm{~J} \mathrm{~cm}^{-2}\right)$; furthermore, for $P$ above this value $d$ gradually increases with $T_{\mathrm{s}}$ and the higher the energy density the higher the factor of $T_{\mathrm{s}}$-induced increase is. The change in substrate temperature from 673 to $748 \mathrm{~K}$ results in an increase in $d$ by $\sim 80 \%$ when $P=3.06 \mathrm{~J} \mathrm{~cm}^{-2} ; d$ increases by a factor more than 2 for the highest value of laser energy density used $\left(3.46 \mathrm{~J} \mathrm{~cm}^{-2}\right)$. It can be concluded that the higher the laser power, the stronger the effect of $T_{\mathrm{s}}$ is. Obviously, heating of the substrate stimulates reactions on the wafer surface; however, the process of oxidation is mainly controlled by laser action. The effect of substrate temperature has a second-order effect for the range of $T_{\mathrm{s}}$ used here. Considering the needs of modern microelectronics, one can conclude that the lower beam energy density around the threshold value, independent of the substrate temperature, gives the best laser oxidized films in terms of low enough thickness and high enough thickness uniformity. This is illustrated, for example, in figure $2(a)$ for $T_{\mathrm{s}}=748 \mathrm{~K}$.

Finally, it should be emphasized that the physics of laser-assisted oxidation is still speculative. Especially, the results presented here cannot answer the question of what the mechanism of laser oxidation itself is. In order to understand the exact process of oxide growth, much more information is needed concerning both the active oxidizing particles created in the oxygen atmosphere under the action of the laser beam and wafer surface reactions. It is clear that more detailed experimental effort is necessary to clarify the effect of laser irradiation on the oxide growth process. We should keep in mind, however, that the photons' energy used here cannot crack the oxygen molecule into two extremely reactive oxygen atoms because the bond energy of $\mathrm{O}_{2}$ is known to be close to $5.1 \mathrm{eV}$. The laser beam may further either react instantly with the surface of the wafer or alternatively with other $\mathrm{O}_{2}$ to form a number of powerful oxidants. We are inclined to assume that the active oxidizing particles in our case are 

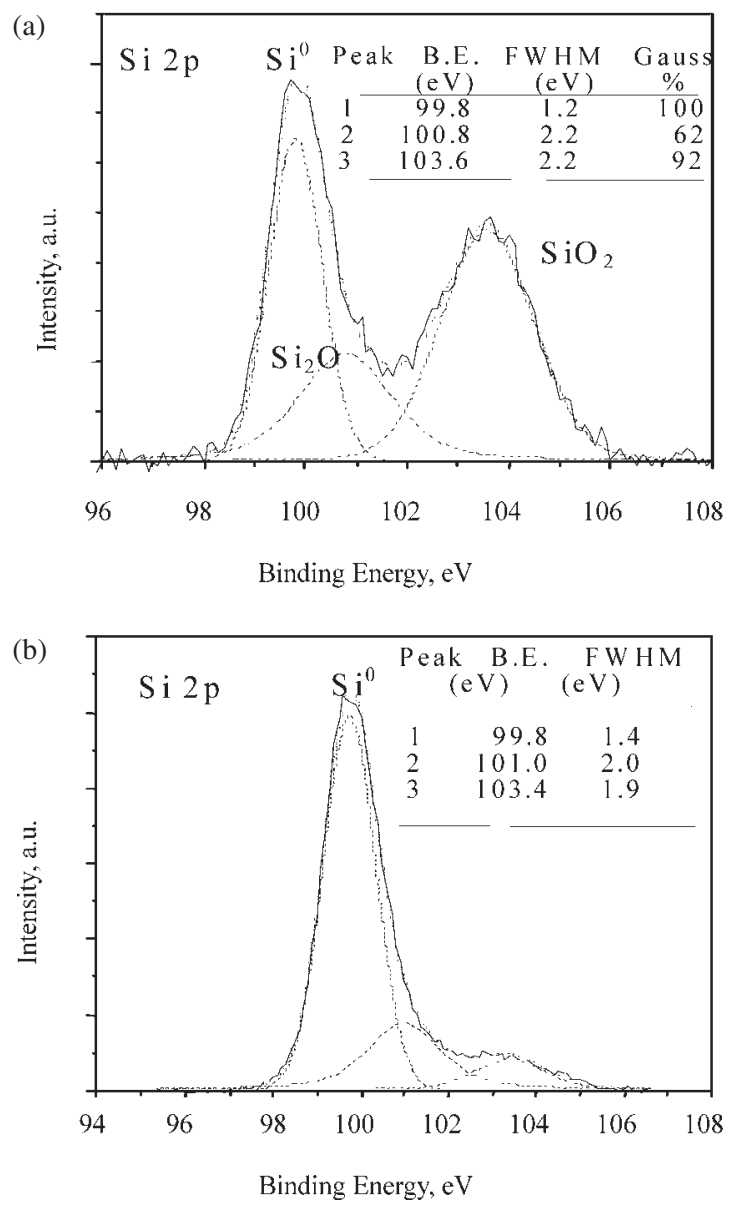

Figure 5. Si $2 p$ spectra of $8 \mathrm{~nm}$ layer oxidized at $T_{\mathrm{s}}=673 \mathrm{~K}$ and laser beam energy density $3.31 \mathrm{~J} \mathrm{~cm}^{-2}:(a)$ at the surface of the layer, (b) at the interface with $\mathrm{Si}(-$, as-recorded data; $\cdots \cdots$, deconvoluted spectra).

oxygen molecules. Most probably, the laser beam induced charged species also contribute to the process of oxidation. For example, it is important to establish the eventual presence of negatively charged species of oxygen, which are known to play a key role in the oxidation of $\mathrm{Si}$. Considering the presence of native oxide on the $\mathrm{Si}$ wafer before laser action, i.e. the fact that $\mathrm{Si}-\mathrm{O}$ bonds are already formed, even though only imperfectly, we assume that the oxide grows by diffusion of an oxygen in molecular form to the interface with $\mathrm{Si}$ /oxide where chemical reactions take place.

\subsection{XPS analysis}

Figure 5 illustrates $\mathrm{Si} 2 \mathrm{p}$ spectra of layers obtained at $T_{\mathrm{s}}=$ $673 \mathrm{~K}$ and a beam energy density of $3.31 \mathrm{~J} \mathrm{~cm}^{-2} ; d=8 \mathrm{~nm}$. The peak positions extracted from the spectra are inserted in the figure. At the surface (figure 5(a)), the spectrum was decomposed into the high binding energy line located at $103.6 \mathrm{eV}$ (referred to as $\mathrm{Si}_{2 \mathrm{p}}^{\text {oxide }}$ ), which is associated with $\mathrm{SiO}_{2}$ and the low binding energy line at $99.8 \mathrm{eV}$ (referred to as $\mathrm{Si}_{2 \mathrm{p}}^{\mathrm{Si}}$ ) originating from the $\mathrm{Si}$ substrate. Both peaks are placed as symmetrical broad lines with FWHM values of $2.2 \mathrm{eV}$ and $1.2 \mathrm{eV}$, respectively. The deconvolution of the $\mathrm{Si}_{2 \mathrm{p}}^{\text {oxide }}$ spectrum results in two peaks, which are attributed to the $\mathrm{SiO}_{2}$ and

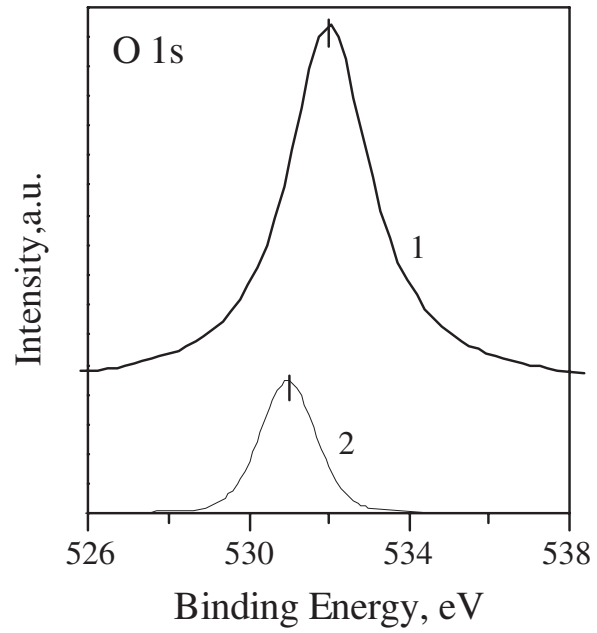

Figure 6. O 1s spectra of the same sample shown in figure 5, (1) at the surface of the layer, FWHM $=2.5 \mathrm{eV}$; (2) at the interface with $\mathrm{Si}, \mathrm{FWHM}=1.6 \mathrm{eV}$.

another phase, namely the intermediate oxidation state, $\mathrm{Si}_{2} \mathrm{O}$. The peak corresponding to $\mathrm{Si}_{2} \mathrm{O}$ is seen as a symmetrical line at $100.8 \mathrm{eV}$ with a FWHM $=2.2 \mathrm{eV}$ (Gaussian 62\%). So, three peaks, elemental $\mathrm{Si},\left(\mathrm{Si}^{0}\right)$, stoichiometric $\mathrm{SiO}_{2}$ and a suboxide $\left(\mathrm{Si}_{2} \mathrm{O}\right)$, are present in the spectrum and the sum of these peaks gives a spectrum equal to the experimental one. As the sputtering approaches the interface with the substrate (figure $5(b)$ ), the intensities of $\mathrm{Si}_{2 \mathrm{p}}^{\text {oxide }}$ and $\mathrm{Si}_{2 \mathrm{p}}^{\mathrm{Si}}$ peaks change but a higher intensity of the $\mathrm{Si}^{0}$ peak is detected for all sputtering times including at the surface. Just at the interface, the spectrum produces the same two components of the $\mathrm{Si}_{2 \mathrm{p}}^{\text {oxide }}$ line $\left(\mathrm{SiO}_{2}\right.$ and $\left.\mathrm{Si}_{2} \mathrm{O}\right)$ in addition to the strong signal from the silicon crystal. The relative ratio of the peak intensities is, however, changed: the intensity of the $\mathrm{SiO}_{2}$ feature is reduced and becomes smaller (by a factor of 2) to that of $\mathrm{Si}_{2} \mathrm{O}$, and about 10 times smaller than the $\mathrm{Si}^{0}$ intensity. The results show that the oxide obtained is nonstoichiometric and consists of $\mathrm{SiO}_{2}$ and $\mathrm{Si}_{2} \mathrm{O}$ states, indicating the presence of excess $\mathrm{Si}$ throughout the whole oxide. The other intermediate oxidation states of $\mathrm{Si}$, namely $\mathrm{SiO}$ and $\mathrm{Si}_{2} \mathrm{O}_{3}$ were not observed. The analysis of the evolution of the $\mathrm{O} 1 \mathrm{~s}$ peak with sputtering times shows the following (figure 6). The $\mathrm{O} 1 \mathrm{~s}$ signal is recorded at $\sim 532 \mathrm{eV}$ at the surface of the layer, which is really the expected position characteristic of $\mathrm{SiO}_{2}$. When thinning the oxide by sputtering, the $\mathrm{O} 1 \mathrm{~s}$ peak shifts to lower energies indicating that the oxidation state of $\mathrm{Si}$ has been reduced. At the interface the $\mathrm{O} 1 \mathrm{~s}$ line is fitted only with a small peak of the elemental oxygen located at $531 \mathrm{eV}$. In summary, XPS analysis illustrates that the oxide obtained is imperfect, nonstoichiometric and nonuniform through the depth-to a first approximation it is predominantly $\mathrm{SiO}_{2}$ at the surface, and the contribution of $\mathrm{Si}_{2} \mathrm{O}$ dominates close to the interface. Obviously, additional post-oxidation technological steps are necessary to improve the microstructure of the laser-oxidized films.

\subsection{Dielectric and electrical properties}

In this section, we give representative results of some oxide and interface parameters of a film obtained at one of the laser treatment regimes used. The layer grown at $673 \mathrm{~K}$ and 


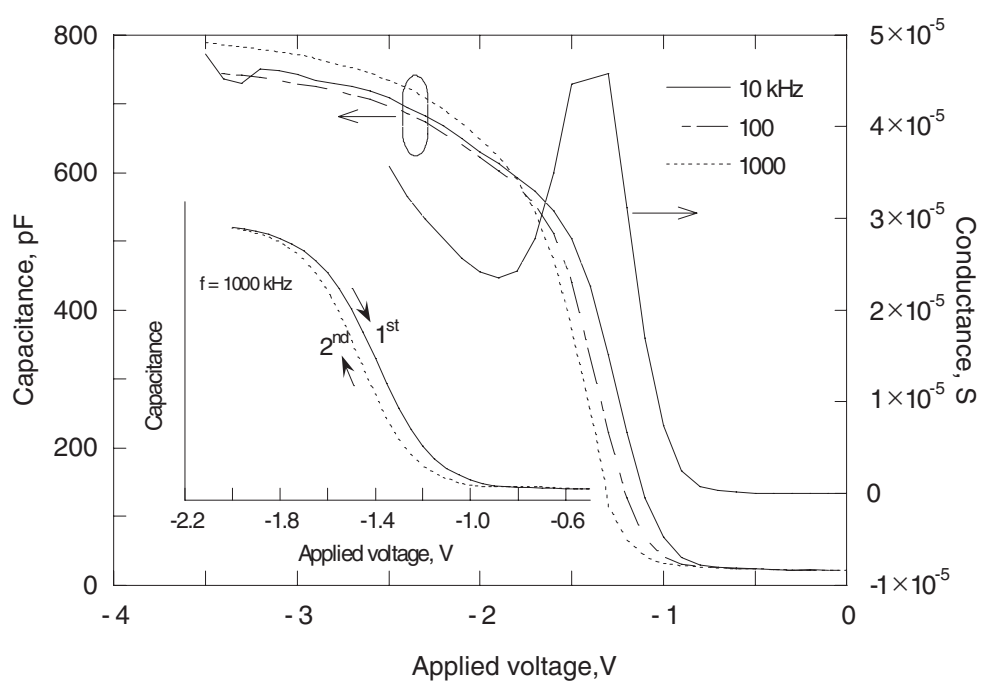

Figure 7. $C-V$ curves measured at different frequencies $(10,100,1000 \mathrm{kHz})$ and $G-V$ curve at $100 \mathrm{kHz}$. The inset shows the hysteresis for the capacitance with respect to applied voltages.

$3.31 \mathrm{~J} \mathrm{~cm}^{-2}$ is selected for a detailed study. To avoid errors due to the nonuniformity of the thickness, an area near the centre of the oxidized region is chosen. For simplicity, we will refer to the film obtained as $\mathrm{SiO}_{2}$ in the following discussions. Figure 7 shows typical high-frequency $C-V$ curves $(10 \mathrm{kHz}$; $100 \mathrm{kHz} ; 1 \mathrm{MHz}$ ) for a MOS capacitor fabricated with an $1.96 \times 10^{-3} \mathrm{~cm}^{2}$ device area on an $8 \mathrm{~nm}$ thick $\mathrm{SiO}_{2}$ film. It can be clearly seen that the film exhibits characteristic MOS behaviour represented by regions of accumulation, depletion and inversion. The flat band voltage, $V_{\mathrm{fb}}$, as derived from the $1 \mathrm{MHz} C-V$ curve is negative, indicating the expected presence of positive oxide charges. $V_{\mathrm{fb}}$ was found to be $-1.3 \mathrm{~V}$ (work function difference between $\mathrm{Al}$ gate and $\mathrm{Si}$ is $-0.7 \mathrm{eV}$ ), giving an oxide charge of $Q_{\mathrm{f}}=1.5 \times 10^{12} \mathrm{~cm}^{-2}$, which is over two orders of magnitude higher than that usually obtained in high quality thermal $\mathrm{SiO}_{2}$. The high value of $Q_{\mathrm{f}}$ is generally associated with structural imperfections, bond defects and poor oxidation process. As was detected by XPS analysis, suboxide is indeed present in the films. This intermediate oxidation state of $\mathrm{Si}$, as well as various microstructural defects manifest as electrically active centres in the form of oxide charges. Further studies are necessary, however, to clarify the nature of the oxide charge in the laser-assisted $\mathrm{SiO}_{2}$ layer grown.

The dielectric constant $\varepsilon$ of the film was estimated to be 3.64 by using the capacitance value in the accumulation mode at $1 \mathrm{MHz}, C_{0}(-3.5 \mathrm{~V})$ and the known oxide thickness. The index of refraction, $n$, according to ellipsometrical measurements is 1.35 . The $\varepsilon$ and $n$ values which are lower than those for the thermal $\mathrm{SiO}_{2}$ can be attributed to the detected $\mathrm{Si}_{2} \mathrm{O}$ in the film, i.e. to poor oxygen stoichiometry.

The film exhibits $C-V$ hysteresis (inset of figure 7) on the lower voltage side of the curves, when the gate voltage is swept from accumulation to inversion and back. This indicates the presence of slow (border) states located very close to the Si/oxide interface and recharge during the back bias cycle. The hysteresis of the $C-V$ curves is negative where the flat band voltage shift arising from the hysteresis effect is as low as $-65 \mathrm{mV}$, and the density of slow states is estimated to be $1.6 \times 10^{11} \mathrm{~cm}^{-2}$. This means that some charge trapping exists in the interfacial region at the Si substrate. Note that slow states usually exist in very thin and ultrathin layers even in high quality thermally grown $\mathrm{SiO}_{2}$. In this sense, the slow states detected here are not a peculiarity of the laser-induced oxidation. The origin of slow states is generally related to microstructural defects, most probably imperfect $\mathrm{Si}-\mathrm{O}$ bonds. It can also be seen from figure 7 that the accumulation capacitance $C_{0}$ decreases slightly from 789 at $1 \mathrm{MHz}$ to about $745 \mathrm{pF}$ at both 10 and $100 \mathrm{kHz}$. The absence of well-pronounced saturation of the curves and the non-ideality feature observed in the range of the accumulation mode is due to the detected leakage current in the film. However, the frequency dispersion of $C_{0}$ is weak, suggesting a small leakage layer. The frequency dispersion is also observed in the depletion region of $C-V$ curves (figure 7). Its value between $10 \mathrm{kHz}$ and $1 \mathrm{MHz}$ near the flat band conditions is $\sim 180 \mathrm{mV}$. This value implies that the interfacial defect charges corresponding to the given frequency ranges are as small as $\sim 4.5 \times 10^{11} \mathrm{~cm}^{-2}\left(C_{0}=4 \times 10^{-7} \mathrm{~F} \mathrm{~cm}^{-2}\right)$. For illustration, the $G-V$ dependence at $100 \mathrm{kHz}$ is shown in figure 7 . The conductivity peak related to the interface states is seen from this curve. The presence of this peak again indicates a low leakage condition and hence a relatively good layer quality for device applications.

The leakage current, $I$, through the capacitor as a function of applied voltage is shown in figure 8 . The current monotonically increases from $10^{-9} \mathrm{~A} \mathrm{~cm}^{-2}$ at very small applied voltages and reaches $10^{-6} \mathrm{~A} \mathrm{~cm}^{-2}$ at an applied field of $\sim 1.2 \mathrm{MV} \mathrm{cm}^{-1}$ for forward bias (negative applied voltage, the electrons are injected from the $\mathrm{Al}$ gate). The shape of the $I-V$ curve for forward bias gives some indication for the appearance of soft breakdowns originating from the defects in the form of oxide charges detected in high density. Well-defined catastrophic (hard) breakdown is not detected up to $-1.5 \mathrm{~V}$ (our experimental limit). The gradual increase of the current up to $\sim 10^{-5} \mathrm{~A} \mathrm{~cm}^{-2}$ at $1.5 \mathrm{~V}$ is also observed at reversed bias (positive applied voltage when the electrons are injected from $\mathrm{Si}$ ), with a change in the slope of the curve (getting smaller) at $\sim 0.6 \mathrm{MV} \mathrm{cm}^{-1}$. A deeper understanding of the electrical 


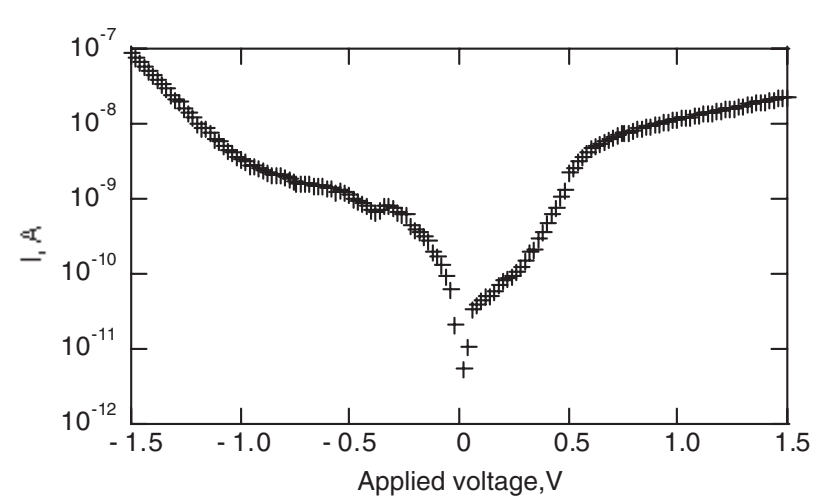

Figure 8. $I-V$ curves of MOS capacitor with oxide obtained at $T_{\mathrm{s}}=673 \mathrm{~K}$ and $3.31 \mathrm{~J} \mathrm{~cm}^{-2} ; d=8 \mathrm{~nm}$.

behaviour of laser-induced oxidized layers, closely related to the growth conditions (including post-oxidation annealing), will be the subject of a future study.

\section{Conclusion}

The results presented in this work allow us to draw the following conclusions. First, amorphous $\mathrm{SiO}_{2}$ thin films can successfully be obtained by pulsed $\mathrm{Nd}$ : YAG laser in $\mathrm{O}_{2}$ ambient at relatively low temperatures. The thickness of the oxide can be monitored easily with respect to the two essential parameters governing the laser oxidation: the laser beam energy density and the substrate temperature. The data suggest that the most desirable growth situation with respect to thicker oxides is to oxidize as close as possible to the laser power corresponding to surface melting without crossing it. As-grown oxide is imperfect and under-stoichiometric, consisting of $\mathrm{SiO}_{2}$ and the suboxide $\mathrm{Si}_{2} \mathrm{O}$ - the amount of $\mathrm{Si}_{2} \mathrm{O}$ increases close to the interface with $\mathrm{Si}$. At the same time, however, the dielectric measurements in the MOS configuration indicate that the laser-assisted oxide generally has parameters close to those of thermally grown $\mathrm{SiO}_{2}$, such as interfacial defect charges, slow state density, leakage current, and the capacitors showed true MOS behaviour. The relatively high density of oxide charges is most probably due to the imperfect microstructure of the oxide and the presence of excess Si, as detected by XPS. Furthermore, the oxide and interface properties could be improved further by appropriate post-oxidation annealing (work is being done on this), which is common practice for $\mathrm{Si}-\mathrm{SiO}_{2}$ devices. Considering the demands of modern microelectronics one can conclude that the Nd: YAG laser oxidation is a promising technique for the formation of small-spot oxide with the desired dimensions and good oxide quality. Although we do not yet understand the oxidation mechanism exactly and the results raise many questions about the growth process, Nd: YAG laser oxidation was shown to produce good quality oxide layers for device applications.

\section{Acknowledgments}

The authors acknowledge partial financial support from TUBITAK (Scientific and Technical Council of Turkey), under project number TBAG/U68, and the Bulgarian National Science Foundation under Contract F-901. Also, LO acknowledges the support from the Turkish Academy of Sciences in the framework of a Young Scientist Award (LO/TUBA-GEBIP/2002-1-17).

\section{References}

[1] Plummer J D, Deal M D and Griffin P B 2000 Silicon VLSI Technology (Englewood Cliffs, NJ: Printice-Hall)

[2] Atanassova E and Dimitrova T 2001 Handbook of Surfaces and Interfaces of Materials vol 4, ed H S Nalwa (San Diego, California, USA: Academic) pp 439-79

[3] Kingon A I, Mana J P and Sbreiffer S K 2000 Nature 4061032

[4] Wilk G D, Wallace R M and Anthony J M 2001 J. Appl. Phys. 895243

[5] Legrand B and Stievenard D 1999 Appl. Phys. Lett. 744049

[6] Boyd I W 1987 Laser Processing of Thin Films and Microstructures (Berlin: Springer) chapter 2

[7] Boyd I W 1983 Appl. Phys. Lett. 42728

[8] Boyd I W and Wilson J I B 1983 Physica B 117 \& 1881030

[9] Cros A and Salvan F 1982 Appl. Phys. A 28241

[10] Orlowski T E and Richter H 1984 Appl. Phys. Lett. 45241

[11] Lavisse L, Grevey D, Langlade C and Vannes B 2002 Appl. Surf. Sci. 186150

[12] Craciun V, Bassim N, Singh R K, Craciun D, Hermann J and Boulmer-Leborgne C 2002 Appl. Surf. Sci. 186288

[13] Baeri P and Rimini E 1996 Mater. Chem. Phys. 46169

[14] Bauerle D 2000 Laser Processing and Chemistry 3rd edn (part II) (Berlin: Springer) 